\title{
Etude de la stabilité d'ensemble des rideaux de soutènement ancrés
}

\author{
Abdul-Hamid Soubra * - Richard Kastner ** \\ Farimah Masrouri *** — Pierre Regenass *
}

* Laboratoire d'Etudes et de Recherches en Génie Civil, ENSAIS

24 boulevard de la Victoire

67084 Strasbourg cedex

\{Ahamid.Soubra, Pierre.Regenass\}@ensais.u-strasbg.fr

** URGC Géotechnique, INSA de Lyon

20 avenue Albert Einstein

69621 Villeurbanne cedex

kastner@gcu-geot.insa-lyon.fr

*** Laboratoire Environnement Géotechnique \& Ouvrages, ENSG
BP 40,54501 Vandouvre-lès-Nancy cedex
masrouri@ensg.u-nancy.fr

RÉSUMÉ. Des essais de laboratoire sur un modèle réduit bidimensionnel ont été effectués afin d'étudier le champ de déplacement d'un soutènement ancré. Une confrontation critique des résultats du modèle réduit aux approches de type Kranz nous conduit à proposer un autre schéma de calcul basé sur la théorie de l'analyse limite en considérant un mécanisme de rupture translationnel. Ce mécanisme est composé de deux blocs rigides dans la zone active et d'un mécanisme de rupture en "log-sandwich "dans la zone passive. La confrontation des résultats numériques avec ceux des expériences réalisées montre la pertinence du mécanisme adopté.

ABSTRACT. Laboratory tests on a two-dimensional model of an anchored sheet pile structure are carried out to study the displacement field of a soil-strcuture system. The comparison of the experimental results with those obtained by the Kranz method shows significant differences. The overall stability analysis of these structures is then investigated by a theoretical model using the upper-bound method of the limit analysis theory. A translational failure mechanism is considered for the calculation scheme. This mechanism is composed of two rigid blocks in the active zone and the so-called log-sandwich mechanism in the passive zone. Numerical results show good agreement with results obtained from laboratory tests.

MOTS-CLÉS : soutènement, ancrage, mesure, calcul, analyse limite.

KEY WORDS: retaining structure, anchor, measurement, calculation, limit analysis. 


\section{Introduction}

L'usage de soutènements flexibles ancrés s'est fortement développé avec la mise au point des techniques de réalisation des tirants injectés en sol meuble. Ces ouvrages remplacent de plus en plus fréquemment les rideaux butonnés qui sont maintenant réservés aux fouilles étroites.

Parmi les multiples facteurs intervenant dans le dimensionnement de l'ancrage, nous nous intéressons ici à la vérification de la longueur du tirant dont l'insuffisance peut provoquer la rupture d'ensemble du rideau et du massif d'ancrage.

Les diverses méthodes proposées pour les tirants superficiels avec plaque d'ancrage, telles que la règle de non interférence entre les prismes de butée devant la plaque d'ancrage et de poussée à l'arrière du rideau se sont vite révélées peu adaptées aux ancrages forés parfois très inclinés sur l'horizontale. Kranz [KRA 53] a proposé une méthode qui, soit sous sa forme originale, soit avec des variantes, est largement utilisée. Dans cette approche, on vérifie que la dimension de l'ancrage (longueur, inclinaison) est suffisante pour que la stabilité du prisme de terre $A B C D$ situé entre le rideau et l'ancrage soit assurée (figure 1). Ce prisme est soumis d'une part à un ensemble de forces tendant à le déstabiliser :

- le poids du prisme $\mathbf{W}$;

- la poussée active de Rankine $P_{a}$ agissant sur la face verticale $B C$;

- l'effort d'ancrage $A$.

D'autre part, les forces concourant à sa stabilisation sont :

- la réaction du rideau $P$ égale à la pression active que le sol exerce sur le rideau ;

- les efforts de frottement et de cohésion $\mathrm{F}$ et $\mathrm{C}$ résultant du cisaillement du sol suivant la ligne de rupture potentielle $C D$.

La stabilité de l'ensemble rideau-ancrage est assurée si la force d'ancrage $A$ ' susceptible de provoquer le glissement du prisme $A B C D$ est supérieure à la force d'ancrage A mobilisée pour assurer la stabilité de l'écran.

Diverses variantes à ce schéma de rupture ont été étudiées [LIT 72], [SCH 76], [OST 77]. Cambefort [CAM 75] a proposé d'adopter une surface potentielle de rupture $\mathrm{CD}$ ayant la forme d'une spirale logarithmique. Sur le même principe, les recommandations TA 86 reprises en 95 [BUR 95], proposent de simplifier l'approche de Cambefort en adoptant une surface de rupture circulaire. Le manque de données expérimentales et l'impossibilité de vérifier ces approches sur ouvrages réels nous ont conduit à réaliser une série d'expérimentations sur un modèle bidimensionnel à rouleaux de Schneebeli.

Après la description succincte du modèle réduit, nous présentons les résultats expérimentaux obtenus à l'aide de ce modèle. Ces résultats sont comparés aux résultats de calcul donnés par la méthode de Kranz. Les écarts importants mis en 
évidence par la confrontation expérience-calcul conduisent à proposer un autre schéma de calcul basé sur la théorie de l'analyse limite en considérant un mécanisme de rupture translationnel. La solution obtenue est une borne supérieure par rapport à la solution exacte pour un matériau de Coulomb $(\varphi, c)$ obéissant au principe du travail plastique maximal de Hill.

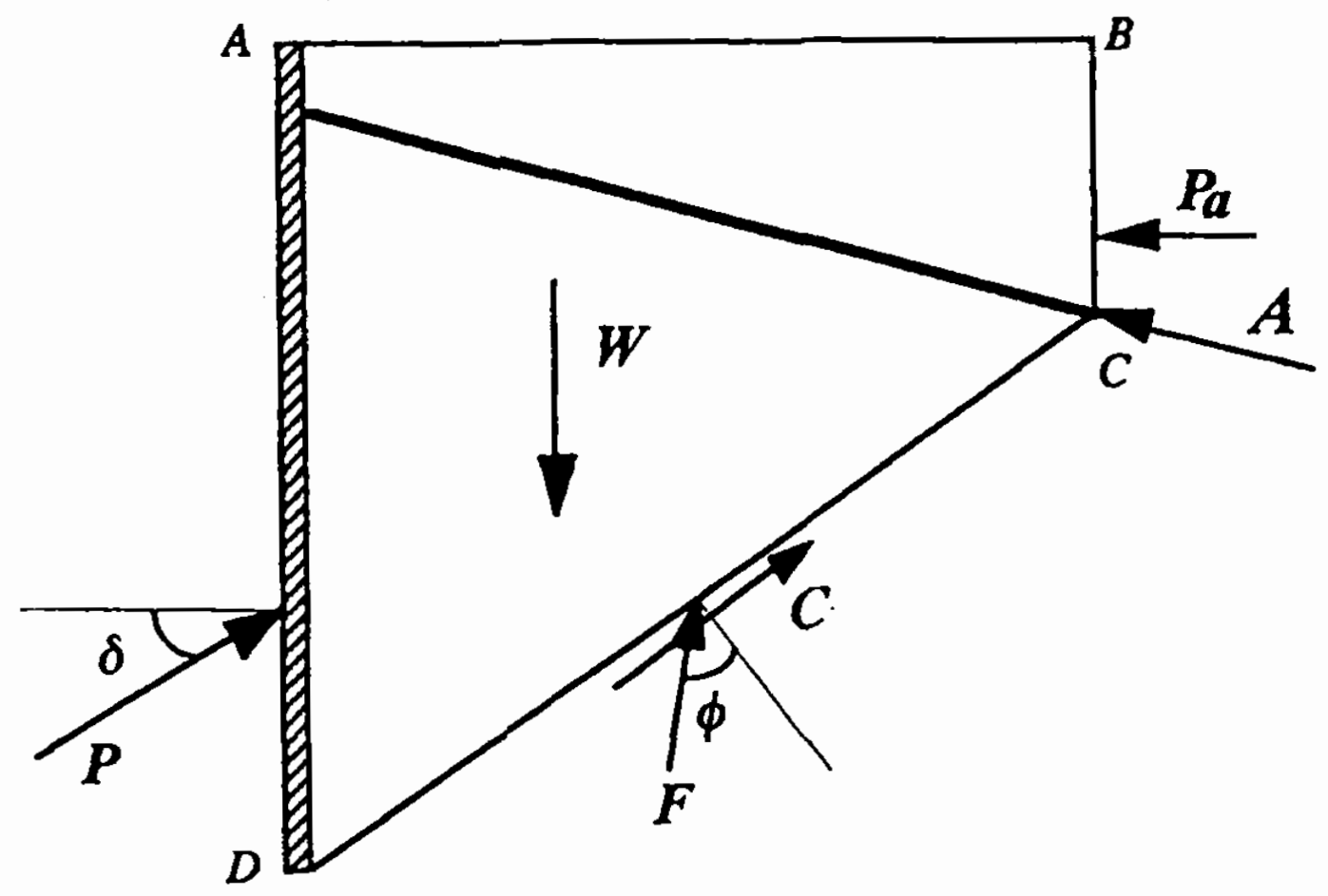

Figure 1. Méthode proposée par Kranz (1953). Method proposed by Kranz

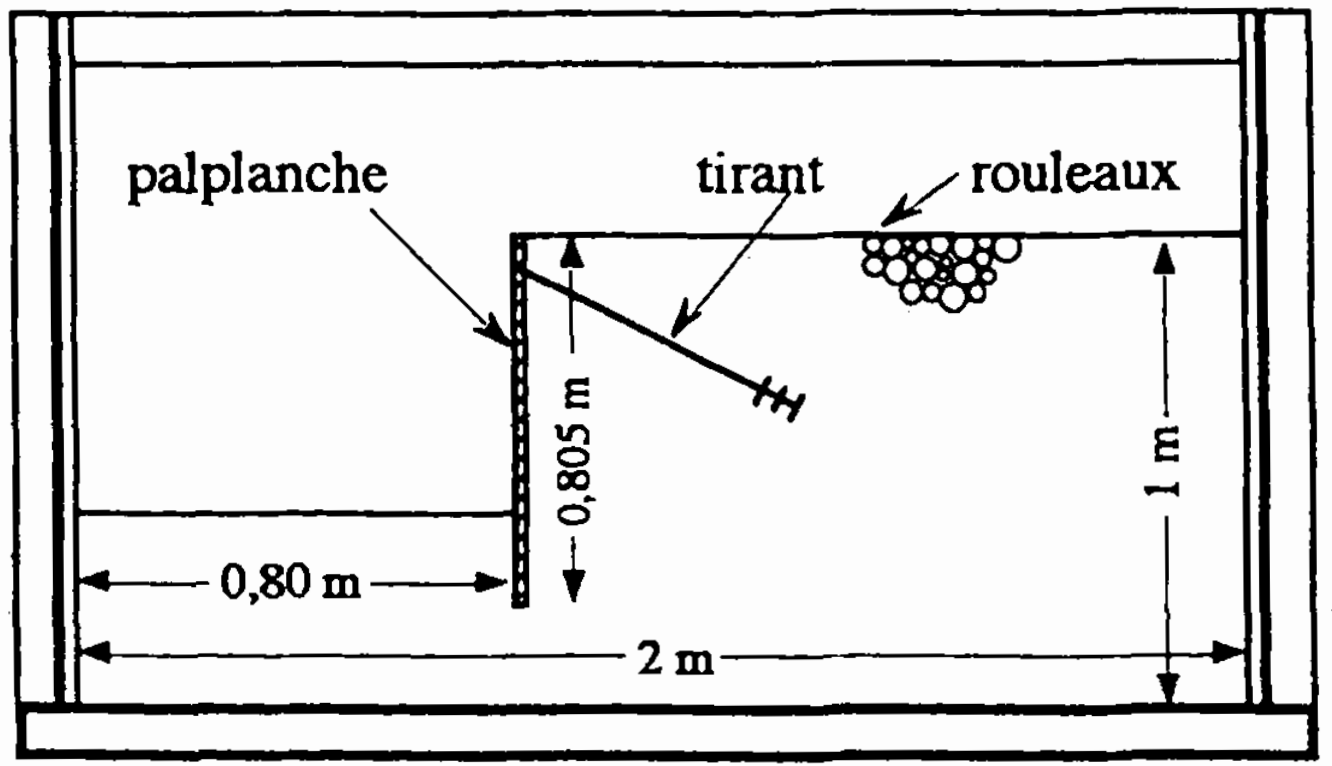

Figure 2. Modèle réduit. Laboratory test 


\section{Modèle réduit}

Le modèle est réalisé à l'aide du matériau analogique de Schneebeli [SCH 57]. Il simule un milieu pulvérulent bidimensionnel, respectant la loi de Coulomb, par un empilement de petits rouleaux cylindriques, parallèles, horizontaux et de même longueur. Dans cette expérimentation, les rouleaux en acier inoxydable ont une longueur de $6 \mathrm{~cm}$ et sont mis en place par couches de $5 \mathrm{~cm}$.

A la suite d'autres expérimentateurs, une combinaison de rouleaux de trois diamètres différents $(3,4$ et $5 \mathrm{~mm})$ est utilisée, afin d'éviter la formation d'assemblages autobloquants constatés lorsqu'on utilise un seul diamètre. Ce matériau analogique bidimensionnel a été caractérisé par des essais de compression biaxiaux, conduisant aux paramètres suivants :

- dẹnsité sèche $\gamma_{d} / \gamma_{w}=6,5$

- angle de frottement interne $\varphi=21^{\circ}$

- angle de dilatance $\psi=5^{\circ}$

- module de compression initial $\mathrm{E}_{\mathrm{i}}=107500 \sqrt{\sigma}[\mathrm{Pa}]$

- coefficient de Poisson $v=0,9$ (matériau bidimensionnel)

Un bâti très rigide et suffisamment grand pour éliminer les effets de bord, supporte cet empilement de rouleaux (figure 2).

Le rideau est simulé par une plaque en Duralumin de $0,805 \mathrm{~m}$ de haut, $80 \mathrm{~mm}$ de large et de $12 \mathrm{~mm}$ d'épaisseur. Ce rideau représente des écrans réels, à l'échelle du dixième au vingtième. Sa raideur relative évaluée suivant les critères définis par Rowe [ROW 72] est du même ordre que celle des soutènements en palplanches ou parois moulées utilisés pour les excavations en site urbain.

La courbure de ce rideau est déterminée par une trentaine de jauges extensométriques collées sur ses deux faces. La pression différentielle du sol sur le rideau est obtenue par double dérivation après lissage du diagramme de courbure expérimental. La reproductibilité de cette détermination est bonne [MAS 86] et conduit à une valeur de la composante normale de la pression active Ka $\gamma$. $\cos \delta=$ 0,39 voisine de celles données par les approches théoriques habituelles. L'angle de frottement sol-écran est compris entre $8^{\circ}$ (mesure par essai de cisaillement direct) et $14^{\circ}$ (mesure de poussée et butée sur un écran vertical en translation).

Le «bulbe " d'ancrage est simulé à l'aide des profilés UPN insérés dans le massif de rouleaux. Ce bulbe est relié à l'écran par deux tiges filetées de $8 \mathrm{~mm}$ de diamètre, placées de part et d'autre du modèle. Ces tiges sont équipées de capteurs mesurant l'effort de traction dans les tirants. Cet ensemble représente un ancrage foré et scellé par injection. 
Le bulbe d'ancrage et le rideau sont insérés séparément dans le massif. Ce n'est qu'après $15 \mathrm{~cm}$ d'excavation que le bulbe sera relié au rideau par des tirants fixés à $5 \mathrm{~cm}$ du sommet, ceci afin de mieux reproduire le déroulement réel des travaux. Par la suite, l'excavation est poursuivie en déblai devant le rideau par couches de $100 \mathrm{~mm}$ jusqu'à la cote de $400 \mathrm{~mm}$; puis, elle est menée par couche de $50 \mathrm{~mm}$ jusqu'à la rupture.

L'étalonnage du système de mesure a montré que les incertitudes sont de l'ordre du pourcent pour les efforts d'ancrage et de quelques pourcents pour la mesure du moment fléchissant par jauges extensométriques. Des essais conduits par deux expérimentateurs différents sur la même configuration ont montré une excellente reproductibilité des essais [KAS 82].

Si la difficulté d'obtenir des données sur ouvrages réels justifie l'utilisation de ce modèle, il convient d'en noter les limites liées en particulier à la faible valeur de $\varphi$. En revanche, la densité sèche élevée du matériau analogique $\left(\gamma_{\delta} / \gamma_{*}=6,5\right)$ permet de limiter l'effet d'échelle lié au niveau de contrainte.

\section{3. Étude expérimentale de la rupture d'ensemble}

Afin d'analyser l'influence des conditions d'ancrage sur la rupture d'ensemble, une campagne d'essais a été entreprise sur le modèle réduit décrit plus haut. Ce travail permet d'étudier le comportement des rideaux ancrés tant dans les conditions de service qu'à la rupture. Les résultats obtenus sur ce modèle ont permis de confirmer et compléter les observations réalisées sur ouvrages réels en service notamment en ce qui concerne les redistributions de la pression active liées à l'effet de voute [KAS 82, MAS 86].

La rupture par manque de fiche avec un rideau butonné, dans des conditions identiques, survient aux alentours de $570 \mathrm{~mm}$ d'excavation. Ainsi une rupture intervenant pour une excavation moindre, peut être attribué à un tirant trop court et être considérée comme une rupture d'ensemble. Cette rupture est confirmée par l'étude du champ de déplacement effectuée par la technique de la stéréophotogrammétrie temporelle [BAC 84]. La figure 3 présente les courbes d'équidéplacement horizontal obtenues par cette méthode pour l'essai A3. Les zones présentant un gradient de déplacement élevé délimitent nettement un schéma de rupture comportant deux zones :

- une zone en état d'équilibre limite de butée devant la fiche ;

- une zone en état d'équilibre limite de poussée derrière l'écran. 


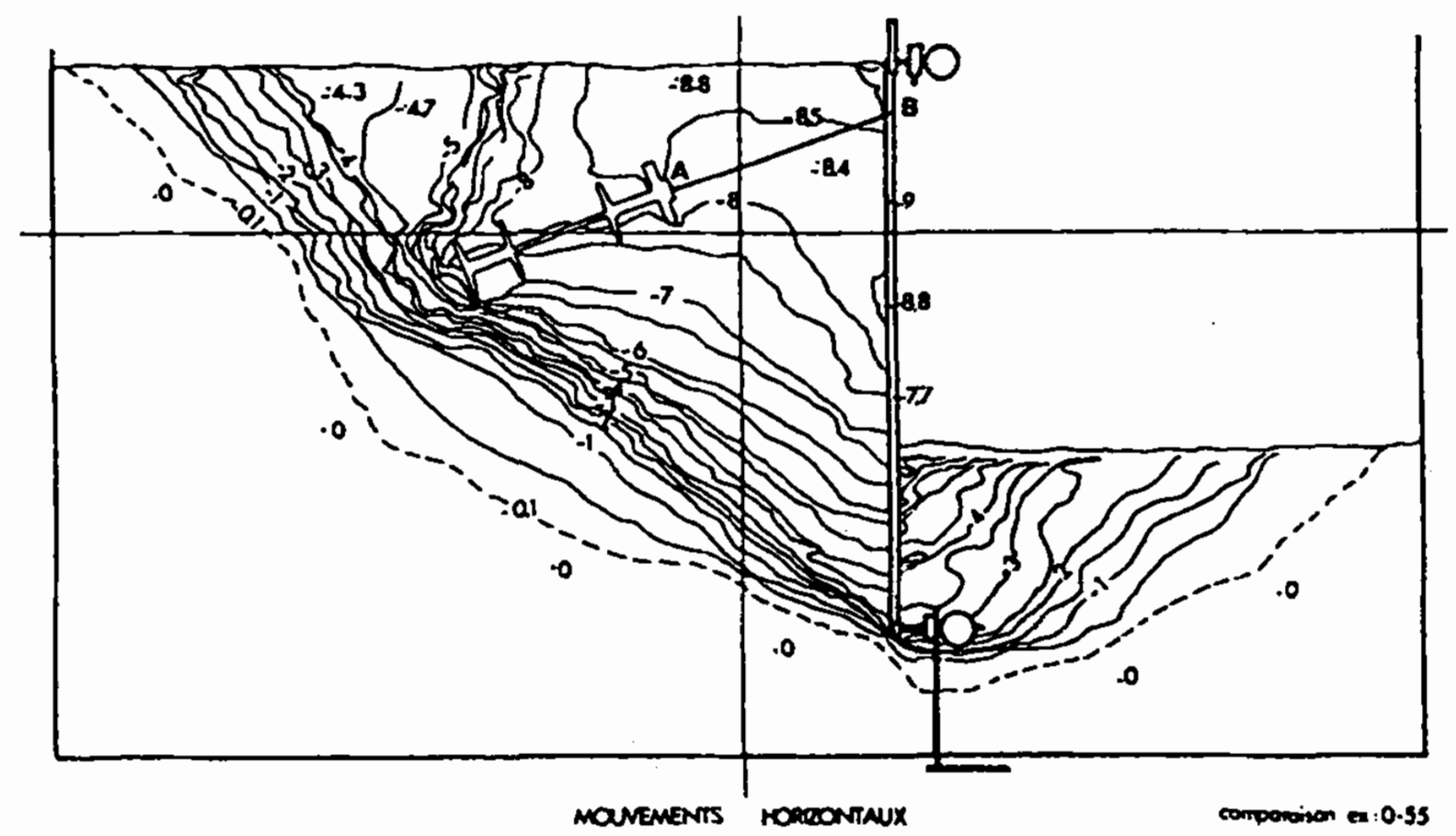

Figure 3. Courbe d'équidéplacement horizontal (mm) obtenues par le traitement stéréophotogrammétrique. Stereophotogrammetric image of horizontal movements (mm)

Le tableau 1 présente l'évolution de la profondeur d'excavation critique lors de la rupture d'ensemble sol-écran, en fonction de l'inclinaison $\alpha$ et de la longueur $1 \mathrm{du}$ tirant.

\begin{tabular}{|c|c|c|c|c|c|}
\hline Essai & $\begin{array}{c}\text { Longueur } \\
\text { de l'ancrage } \\
1(\mathrm{~m})\end{array}$ & $\begin{array}{c}\text { Inclinaison } \\
\text { de l'ancrage } \\
\alpha\left(^{\circ}\right)\end{array}$ & $\begin{array}{c}\text { Profondeur } \\
\text { d'excavation } \\
\text { critique }(\mathrm{m})\end{array}$ & $\begin{array}{c}\text { Effort } \\
\text { d'ancrage } \\
\text { mesuré } \\
(\mathrm{kN} / \mathrm{m})\end{array}$ & $\begin{array}{c}\text { Effort } \\
\text { d'ancrage } \\
\text { calculé } \\
(\mathrm{kN} / \mathrm{m})\end{array}$ \\
\hline A1 & 0,850 & 22 & 0,505 & 2,55 & 2,50 \\
A2 & 0,750 & 22 & 0,490 & 2,90 & 1,10 \\
A3 & 0,650 & 22 & 0,470 & 2,60 & 0,00 \\
A4 & 0,550 & 22 & 0,425 & 2,35 & $-0,60$ \\
A5 & 0,550 & 31 & 0,450 & 2,70 & $-0,60$ \\
A6 & 0,750 & 31 & 0,510 & 3,20 & 2,00 \\
\hline
\end{tabular}

Tableau 1. Résultats de l'ensemble des essais, mesurés et calculés. Calculated and measured results of all model tests 
La rupture apparaissant de manière progressive, la détermination de la profondeur d'excavation à la rupture est entachée d'une marge d'incertitude de l'ordre du centimètre.

Une augmentation logique de la profondeur d'excavation avec la longueur d'ancrage est notée. De même, l'augmentation d'inclinaison de l'ancrage apparaît légèrement bénéfique à la stabilité d'ensemble [MAS 86].

\section{Comparaison mesure-calcul par la méthode de Kranz}

Pour l'ensemble de ces essais, la force d'ancrage susceptible de provoquer la rupture d'ensemble a été calculée par l'approche de Kranz en considérant que ces rideaux de soutènement peu flexibles travaillent suivant le mode ancré simplement buté. Le calcul a été effectué suivant les hypothèses de l'approche de Kranz exposées en introduction. Pour le calcul de la pression active sur l'écran, nous avons retenu un frottement relatif $\delta / \varphi$ égal à $2 / 3$ et un coefficient de poussée normale Kay.cos $\delta$ égal à 0,39 en accord avec les résultats expérimentaux en l'absence d'effet de voûte (rideaux sans ancrage ou avec étaiement de faible raideur):

Si l'on excepte l'essai Al correspondant à l'ancrage le plus long, les résultats présentés dans le tableau 1 montrent que l'effort d'ancrage theorique est toujours nettement inférieur à l'effort réel mesuré sur le modèle au moment de la rupture ; l'effort calculé pouvant être nul voire négatif. A travers ces exemples, il apparaît nettement que l'approche de Kranz ne décrit pas correctement l'effort d'ancrage mobilisé dans le cas d'un ancrage trop court.

Pour tenter d'expliquer ces discordances, l'essai A3 a été plus particulièrement analysé en envisageant l'incidence de différents facteurs.

i. Forme de la surface de rupture

La méthode de Kranz a été comparée au schéma proposé par les règles TA 95, bien que la surface de rupture apparaisse plus proche d'une droite que d'un cercle.

ii. Distribution réelle des contraintes au contact sol-écran de soutènement Dans le calcul selon Kranz, on admet que la répartition des contraintes de poussée au contact sol-écran suit la répartition théorique d'équilibre limite. Les pressions différentielles mesurées dans ces essais ont montré que la contrainte réelle était le plus souvent notablement supérieure à la valeur théorique en raison de l'effet de voûte (figure 4). En effet, lorsque la répartition de contrainte mesurée est prise en compte, la poussée passe alors de $8,2 \mathrm{kN} / \mathrm{m}$ a $9,5 \mathrm{kN} / \mathrm{m}$. Dans ces conditions, l'effort d'ancrage susceptible théoriquement de provoquer une rupture d'ensemble augmente considérablement. Ceci souligne l'une des faiblesses du schéma de calcul proposé par Kranz qui présente une sensibilité excessive à la valeur des efforts de 
poussée au contact sol-écran, alors que les mesures tant sur modèle que sur ouvrages réels indiquent des valeurs et répartitions bien différentes de la pression active théorique, en raison des redistributions d'efforts provoquées par la flexion des rideaux de soutènement.

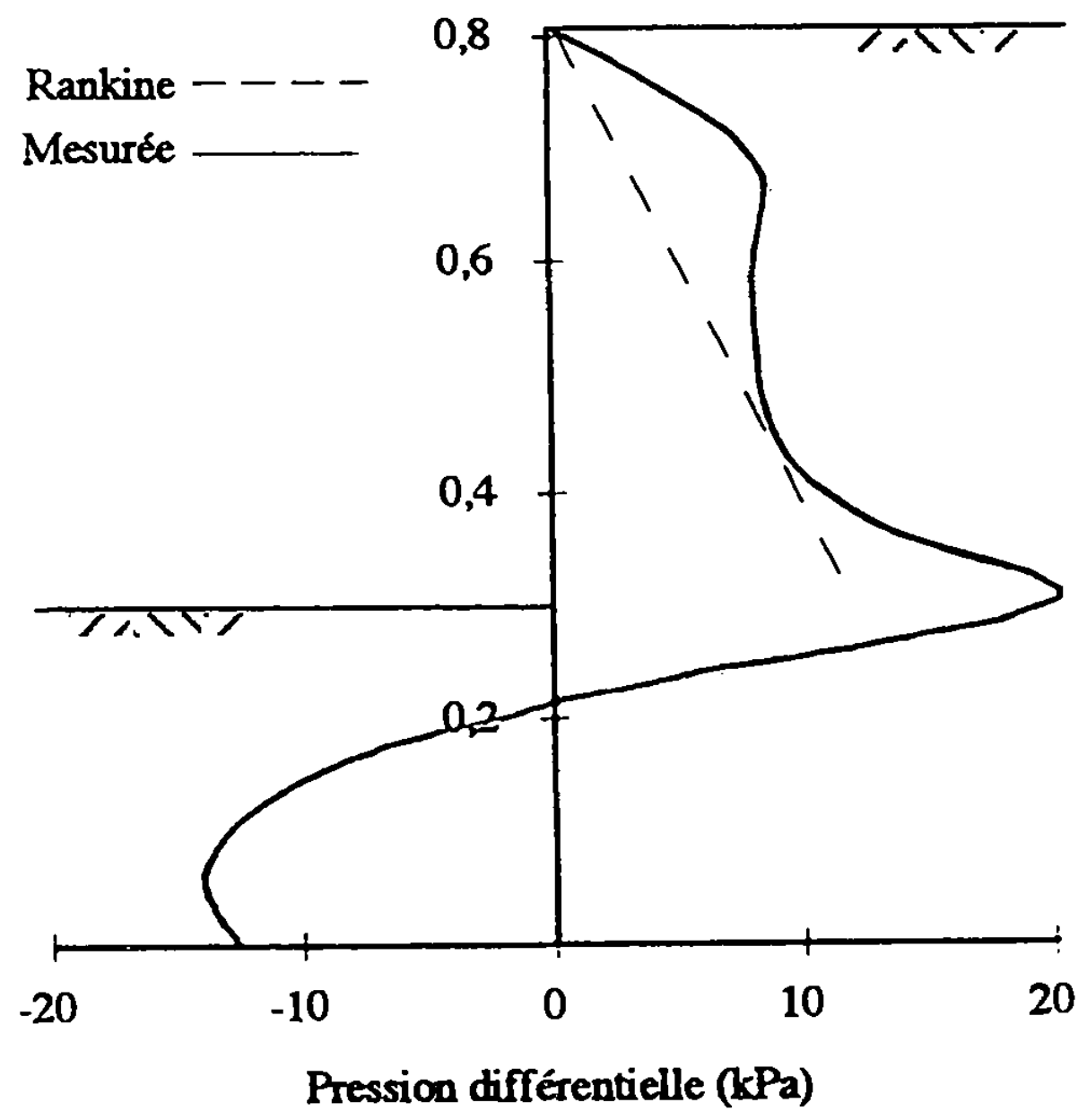

Figure 4. Courbe de pression différentielle de l'essai A3. Net pressure diagram of test A3

iii. Position du point d'effort tranchant nul

Les mesures font apparaître un léger effet d'encastrement qui déplace ce point à $25 \mathrm{~mm}$ du bas de l'écran.

iv. Inclinaison de l'ancrage

Les photos montrent que l'inclinaison réelle de l'ancrage passe de $22^{\circ}$ a $26^{\circ}$ au moment de la rupture.

Le tableau 2 présente l'évolution des efforts calculés pour chacune de ces modifications d'hypothèses séparément, puis l'influence simultanée de l'ensemble de ces facteurs. 
La prise en compte simultanée dans les calculs de chacun de ces paramètres conduit à un effort calculé proche de la valeur mesurée. Il faut noter toutefois que le facteur ayant une influence prépondérante est celui de la poussée au contact sol-écran.

\begin{tabular}{|c|c|c|c|c|c|c|c|}
\cline { 2 - 7 } \multicolumn{1}{c|}{} & Kranz & TA 95 & $\begin{array}{c}\text { Prise en } \\
\text { compte } \\
\text { de la } \\
\text { poussée } \\
\text { mesurée }\end{array}$ & $\begin{array}{c}\text { Position de } \\
\text { point } \\
\text { d'effort } \\
\text { tranchant } \\
\text { nul }\end{array}$ & $\begin{array}{c}\text { Inclinai- } \\
\text { son réelle } \\
\text { de } \\
\text { l'ancrage }\end{array}$ & $\begin{array}{c}\text { Prise en } \\
\text { compte de } \\
\text { l'ensemble } \\
\text { des facteurs }\end{array}$ & $\begin{array}{c}\text { Valeur } \\
\text { expéri- } \\
\text { mentale }\end{array}$ \\
\hline $\begin{array}{c}\text { Force } \\
\text { dans le } \\
\text { tirant } \\
(\mathrm{kN} / \mathrm{m})\end{array}$ & 0 & 0,17 & 1,83 & 0,92 & 0,23 & 2,95 & 2,60 \\
\hline
\end{tabular}

Tableau 2. Facteurs affectant la valeur calculée de la force dans le tirant. Factors influencing the theoretical anchor force

En résumé, la prise en compte de cet ensemble de facteurs non connus a priori, permet, après avoir effectué l'essai, de calculer par la méthode de Kranz un effort dans le tirant tout à fait acceptable. Par contre, l'écart apparaît considérable par rapport au calcul a priori.

Il convient de remarquer ici que le schéma de calcul proposé par Kranz repose sur un découpage arbitraire du massif sollicité par le rideau de soutènement et l'ancrage. Si l'on considère l'ensemble du massif en rupture, mis en évidence par l'étude stéréophotogrammétrique, il apparaît que l'effort d'ancrage est un effort interne. Il est donc plus logique de considérer un schéma de calcul prenant en compte l'ensemble des zones en rupture.

Dans la suite de cette étude, nous proposons un modèle théorique basé sur la méthode cinématique de la théorie de l'analyse limite. Ce modèle permet d'étudier la stabilité de l'ensemble sol-structure à partir du champ de déplacement donné par l'étude stéréophotogrammétrique.

\section{Modèle théorique}

L'étude des problèmes de stabilité par la théorie de l'analyse limite (Méthodes cinématique et statique) a fait l'objet de nombreux travaux. On peut citer entre autres les travaux de [ABD 91, 94, 97], [DEB 89, 91, 93], [PAS 78], [BOT 80], [SAL 90, 93, 95a, 95b].

Dans ce travail, notre choix s'est porté sur l'approche cinématique de la théorie de l'analyse limite. Cette méthode consiste à choisir un mécanisme de rupture cinématiquement admissible respectant les conditions aux limites en vitesses, et à 
égaler la puissance des efforts extérieurs à la puissance dissipée [SAL 83]. Le mécanisme de rupture retenu dans la présente analyse s'inspire des courbes d'équidéplacement observées à la rupture.

\subsection{Hypothèses}

Les hypothèses envisagées dans cette étude sont :

- le sol est homogène et isotrope. Il est caractérisé par son angle de frottement interne $\varphi$, sa cohésion c et son poids volumique $\gamma$. Il est supposé obéir à la règle d'écoulement associée au critère de rupture de Mohr-Coulomb ;

- l'interface sol-écran est caractérisée par un angle de frottement constant $\delta$, ceci est en conformité avec la cinématique envisagée dans cette étude comme on le verra plus loin ;

- l'écran de soutènement et le tirant d'ancrage sont considérés rigides ;

- on admet que la résistance propre de l'ancrage est suffisante ;

- la présence de la nappe phréatique n'est pas envisagée dans cette étude.

\subsection{Choix du mécanisme étudié}

Les résultats expérimentaux ont permis de mettre en évidence une translation quasi horizontale de l'écran. Au moment de la rupture, l'analyse du champ de déplacement par stéréophotogrammétrie révèle la présence de deux zones de rupture : une zone en état actif à l'amont de l'écran et une zone en état passif à l'aval de l'écran (figure 3). La ligne de glissement entre l'extrémité de l'ancrage et le pied de l'écran est quasi rectiligne, il apparaît donc légitime de l'assimiler à une droite. Le schéma de rupture retenu dans l'étude présente se compose donc (figure 5) :

- d'un prisme rigide de forme triangulaire EFG en effondrement, ce prisme s'appuyant sur l'extrémité de l'ancrage $G$. Il est complètement défini par les paramètres angulaires $\theta_{1}$ et $\theta_{2}$;

- d'un prisme rigide en forme de quadrilatère GFAD délimité par l'écran, le bloc précédent et la ligne de glissement GD reliant l'extrémité de l'ancrage au pied de l'écran ;

- d'une zone de rupture en état d'équilibre limite de butée CDHIJ à l'aval de l'écran. Le mécanisme de rupture considéré dans cette zone est un 'log-sandwich'. Il est composé d'une zone à cisaillement radial $\mathrm{CHI}$ comprise entre deux blocs rigides $\mathrm{CDH}$ et $\mathrm{CIJ}$. Ce mécanisme est complètement défini par les paramètres angulaires $\theta_{3}$ et $\theta_{4}$. Le choix de ce mécanisme de rupture n'est pas arbitraire. En effet, Chen [CHE 75] a montré que ce mécanisme donne dans la plupart des cas, la plus petite borne supérieure parmi les six mécanismes qu'il a abordés dans l'étude des pressions passives des terres. 


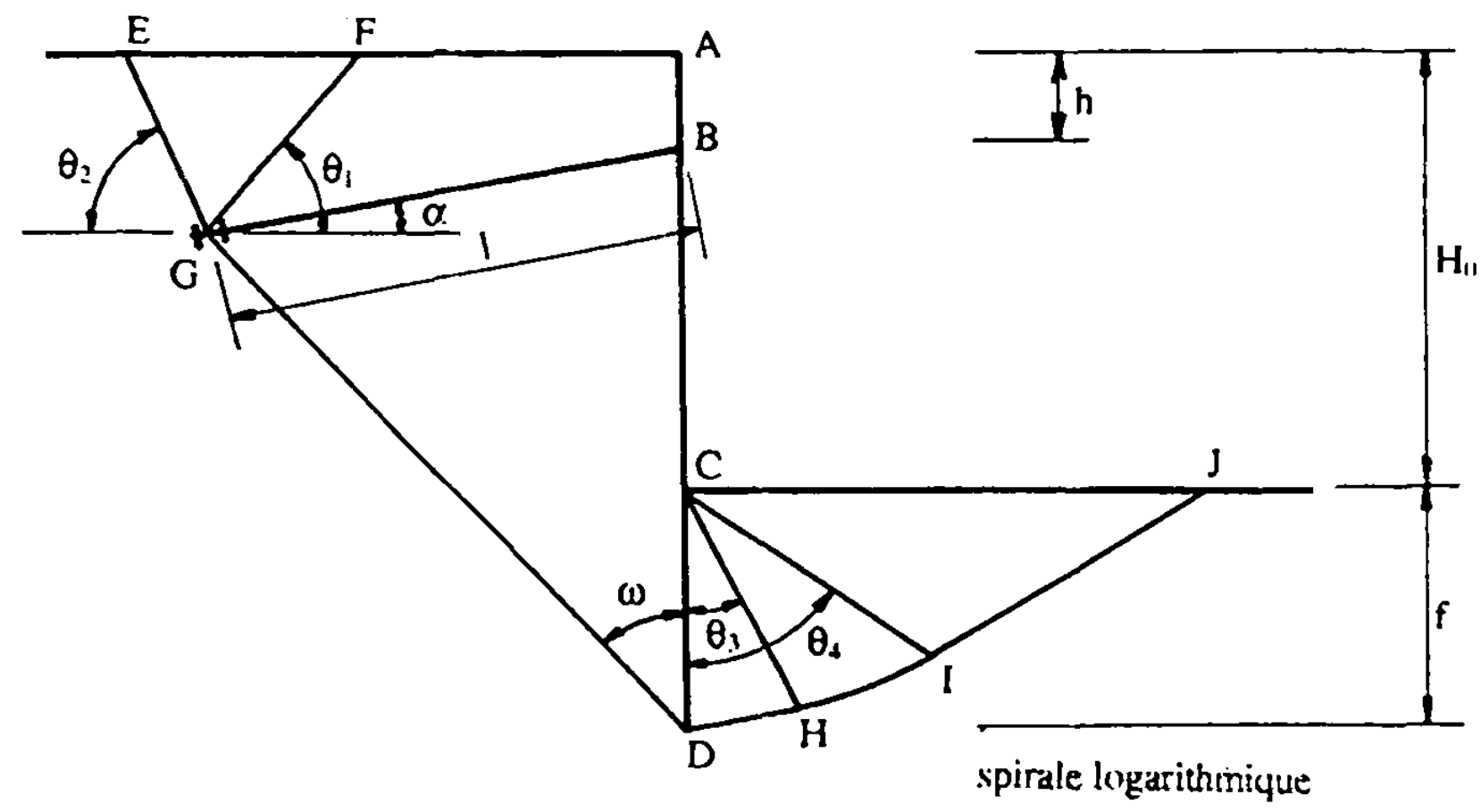

Figure 5. Mécanisme de rupture retenu. Failure mechanism

\subsection{Etude de la cinématique du mécanisme envisagé}

Comme il a été cité plus haut, la méthode cinématique en analyse limite requiert un mécanisme de rupture cinématiquement admissible respectant les conditions aux limites en vitesses. Dans le cas d'un matériau de Coulomb $(\varphi, c)$ parfaitement plastique et respectant la règle de normalité, la condition d'un cisaillement plastique impose que la vitesse de glissement le long d'une surface de discontinuité de vitesse soit inclinée d'un angle $\varphi$ avec cette surface. Dans le cas présent (figure 6), cette condition est vérifiée le long de toutes les surfaces de discontinuité de vitesse sauf au niveau de l'écran où l'on fait l'hypothèse d'une vitesse de discontinuité tangente au rideau. Ainsi, le cisaillement le long de l'interface sol-écran aura lieu sans aucune deformation volumique.

Les hodographes de vitesse des différents blocs en rupture sont représentés sur la figure 7. Dans la zone active, le bloc EFG (respectivement GFAD) se déplace comme un corps rigide avec une vitesse de translation $V_{1}$ (respectivement $V_{2}$ ). Le rideau subit un mouvement de translation horizontale de vitesse $V_{p}$. Quant a la zone passive, les deux blocs rigides de forme triangulaire $\mathrm{CDH}$ et $\mathrm{CIJ}$ se déplacent respectivement avec les vitesses $V_{3}$ et $V_{4}$. La zone à cisaillement radial, délimitée par la spirale logarithmique d'angle $\varphi$, subit un mouvement de translation. 


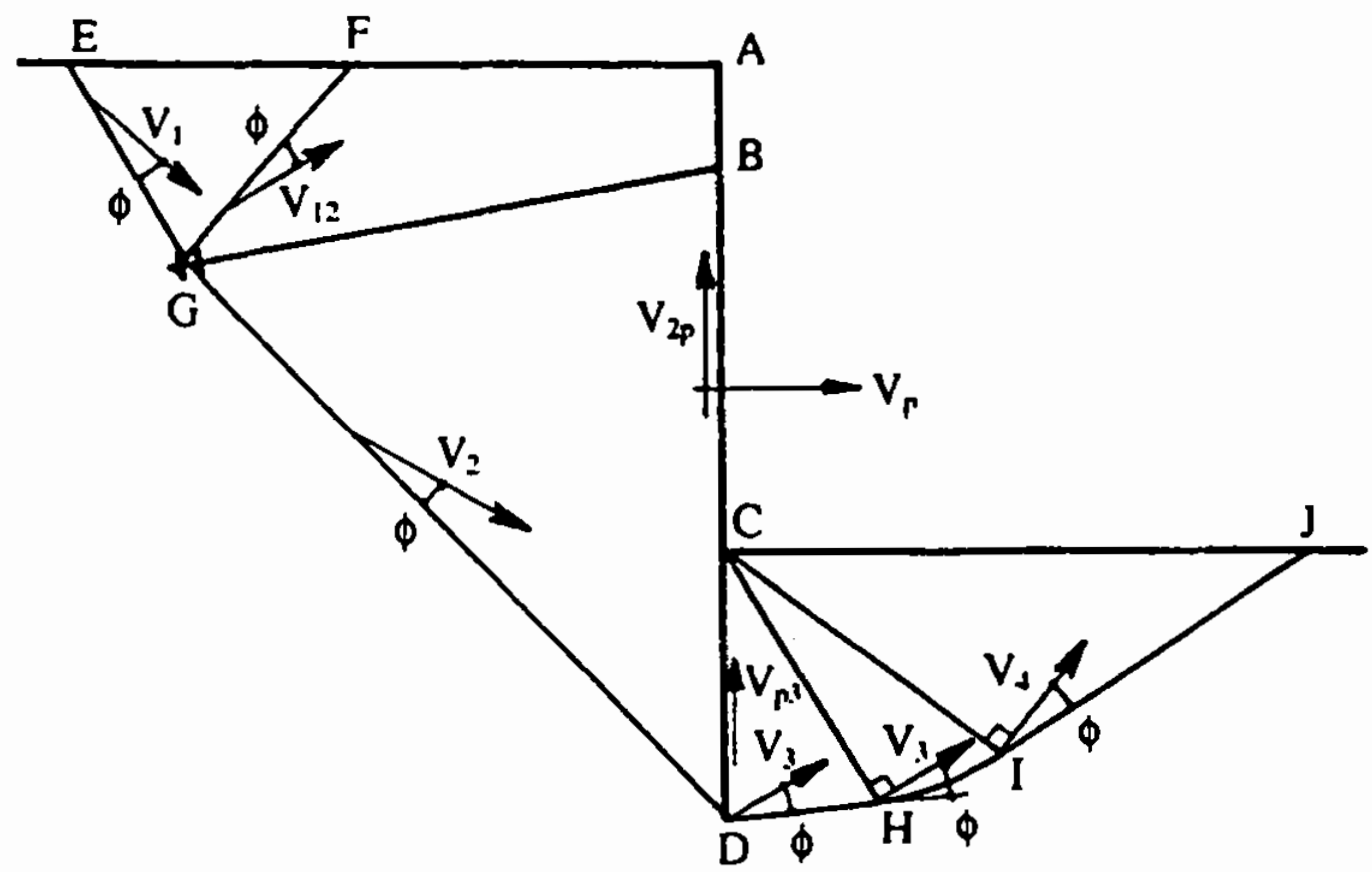

Figure 6. Champ de vitesses du mécanisme de rupture. Velocity field of the failure mechanism
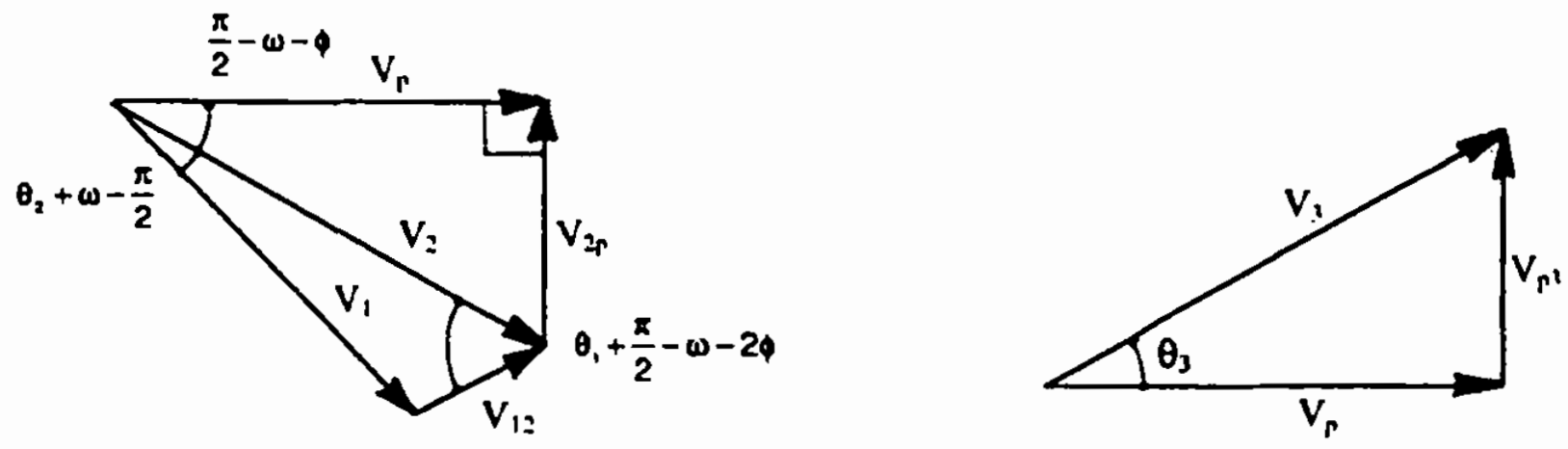

Figure 7. Hodographe des vitesses dans la zone active et dans la zone passive. Velocity hodograph in the active and in the passive zones

Le champ de vitesse le long de la surface HI est décrit par l'équation suivante :

$V(\theta)=V_{3} \exp \left(\theta-\theta_{3}\right) \tan \varphi$

Le champ de vitesse étant complètement défini, l'écriture de l'équation du bilan énergétique du mécanisme étudié fait l'objet du paragraphe suivant. 


\subsection{Bilan énergétique du mécanisme étudié}

Le bilan énergétique consiste à égaler la puissance des efforts extérieurs à la puissance dissipée. Les efforts agissant sur le mécanisme de rupture envisagé sont le poids des différents blocs EFG, GFAD, $\mathrm{CDH}, \mathrm{CHI}$ et $\mathrm{CIJ}$. La puissance dissipée a lieu :

- le long des surfaces de discontinuité de vitesse EG, GF, GD, DH, HI, IJ et le long de toutes les lignes radiales dans la zone en spirale logarithmique ; cette puissance dissipée par unité de surface est donnée par $D=c \Delta V$ où c est la cohésion et $\Delta \mathrm{V}$, la composante tangentielle de la vitesse le long de la surface de discontinuité ;

- le long de l'écran où cette même puissance est donnée par :

$$
D=P_{1} \tan \delta \cdot V_{2 p}+P_{2} \tan \delta \cdot V_{p 3}
$$

$P_{1}$ et $P_{2}$ sont respectivement les forces normales agissant respectivement le long des surfaces $A D$ et $C D$.

La détermination de la puissance dissipée requiert la détermination des efforts $P_{1}$ et $\mathrm{P}_{2}$ définis précédernment (cf. équation [2]). L'effort $\mathrm{P}_{1}$ est déterminé à partir de l'écriture de l'équation du bilan énergétique du massif de sol EGDA à gauche de l'écran. En effet, en égalant la puissance des efforts extérieurs appliqués à ce volume de sol à la puissance dissipée le long des surfaces de discontinuité de vitesses, on obtient l'expression de la force inconnue $P_{1}$ pour laquelle le massif de sol EGDA est en état d'équilibre limite de poussée. Cette force est fonction des seuls paramètres angulaires $\theta_{1}$ et $\theta_{2}$. L'application de la même démarche au volume de sol à droite de l'écran, permet de déterminer l'expression de la deuxième force inconnue $\mathrm{P}_{2}$ pour laquelle le massif de sol $\mathrm{CDHIJ}$ est en état d'équilibre limite de butée. Cette force est fonction des seuls paramètres angulaires $\theta_{3}$ et $\theta_{4}$.

\subsection{Mise en auvre numérique du problème}

Rappelons ici brièvement les différentes étapes de calcul :

- on exprime les énergies résistantes et mobilisatrices qui permettent de formuler le bilan énergétique de toute la masse du sol en rupture (zone active et passive) ;

- on définit la sécurité vis-à-vis de la rupture d'ensemble de deux manières :

1. $\mathrm{F}_{\mathrm{a}}=$ rapport de la contrainte de cisaillement maximale à la contrainte de cisaillement mobilisée $F_{s}=\tau_{\max } / \tau_{\text {mob }}$;

2. $\mathrm{F}_{\mathrm{c}}=$ rapport des énergies résistantes aux énergies dues aux forces motrices. 
Ces deux approches conduisent à deux valeurs différentes du facteur de sécurité sauf au moment de la rupture où $\mathrm{F}_{\mathrm{s}}=\mathrm{F}_{\mathrm{c}}=1$;

- on minimise ces deux facteurs de sécurité par rapport aux quatre paramètres angulaires $\theta_{1}, \theta_{2}, \theta_{3}$ et $\theta_{4}$ définissant le mécanisme de rupture. Nous obtenons ainsi les facteurs de sécurité minimaux pour le mécanisme envisagé. Les valeurs optimales des quatre paramètres angulaires permettent de connaître la position la plus critique de la surface de rupture.

\section{Résultats numériques}

Dans ce paragraphe, nous présentons quelques résultats numériques qui montrent l'influence des différents paramètres tels que la profondeur d'excavation $\mathrm{H}_{0}$, la longueur de l'ancrage 1 et son inclinaison $\alpha$, sur les valeurs du facteur de sécurité ainsi que sur la surface de glissement la plus critique. Les données qui suivent seront conservées dans les deux paragraphes 6.1 et 6.2 :

$$
H_{0}+f=0,805 m ; h=0,05 m ; \varphi=21^{\circ} ; \gamma=65 \mathrm{kN} / \mathrm{m}^{3} ; \delta=8^{\circ}
$$

où $\mathrm{H}_{0}$ est la profondeur d'excavation critique ; $\mathrm{f}$, la fiche correspondante et $\mathrm{h}$, la cote du point d'ancrage sur l'écran.

\subsection{Influence de la profondeur d'excavation}

La figure 8 montre la variation des facteurs de sécurité $F_{s}$ et $F_{c}$ en fonction de la profondeur d'excavation $\mathrm{H}_{0}\left(\mathrm{l}=85 \mathrm{~cm} ; \alpha=22^{\circ}\right)$.

Pour montrer l'influence de la profondeur d'excavation sur la surface de rupture la plus critique, nous présentons sur la figure 9 les surfaces de rupture critiques pour deux valeurs de la profondeur d'excavation $\left(\mathrm{H}_{0}=40 \mathrm{~cm}\right.$ et $\left.53 \mathrm{~cm}\right)$. Dans le cas où $\mathrm{H}_{0}=40 \mathrm{~cm}$, la stabilité d'ensemble est assurée $\left(F_{c}=2,18\right.$ et $\left.F_{s}=1,52\right)$ alors que la valeur $\mathrm{H}_{0}=53 \mathrm{~cm}$ correspond à la situation de la rupture imminente $\left(\mathrm{F}_{\mathrm{s}}=\mathrm{F}_{\mathrm{c}}=1\right)$. La surface de glissement dans la zone active reste identique quelle que soit la profondeur d'excavation $H_{0}$. Les angles $\theta_{1}$ et $\theta_{2}$ obtenus à partir de la minimisation numérique sont tous les deux égaux à $\pi / 4+\varphi / 2$. Quant aux angles $\theta_{3}$ et $\theta_{4}$, ils varient de telle manière que les deux angles $I \hat{C} J$ et $I \hat{J C}$ sont tous les deux égaux à $\pi / 4-\varphi / 2$. Le prisme CIJ est donc en état d'équilibre limite de Rankine. 


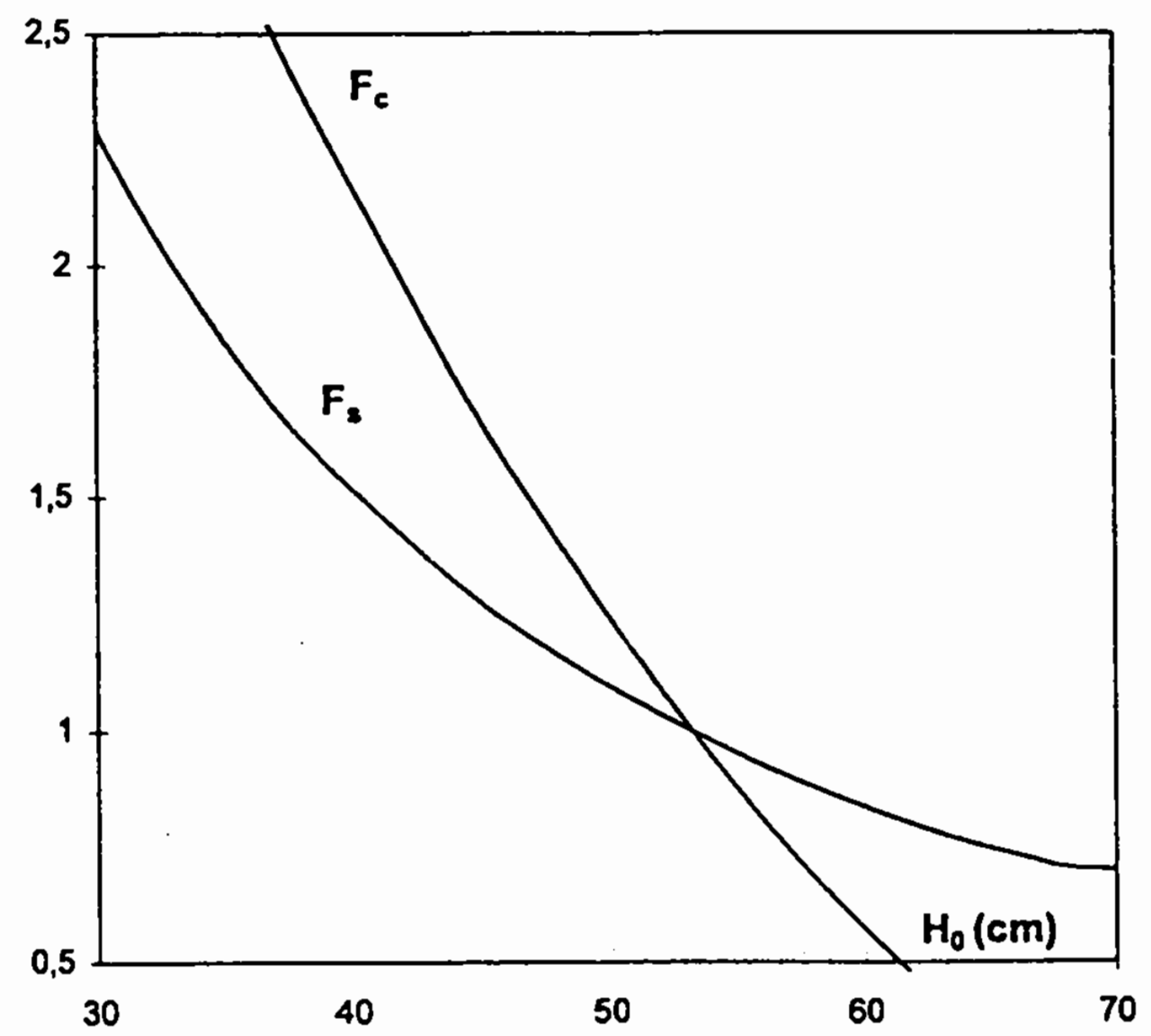

Figure 8. Variation des facteurs de sécurité $F_{s}$ et $F_{c}$ avec la profondeur d'excavation $H_{0} . \mathrm{F}_{\mathrm{s}}$ and $\mathrm{F}_{\mathrm{c}}$ versus excavation depth

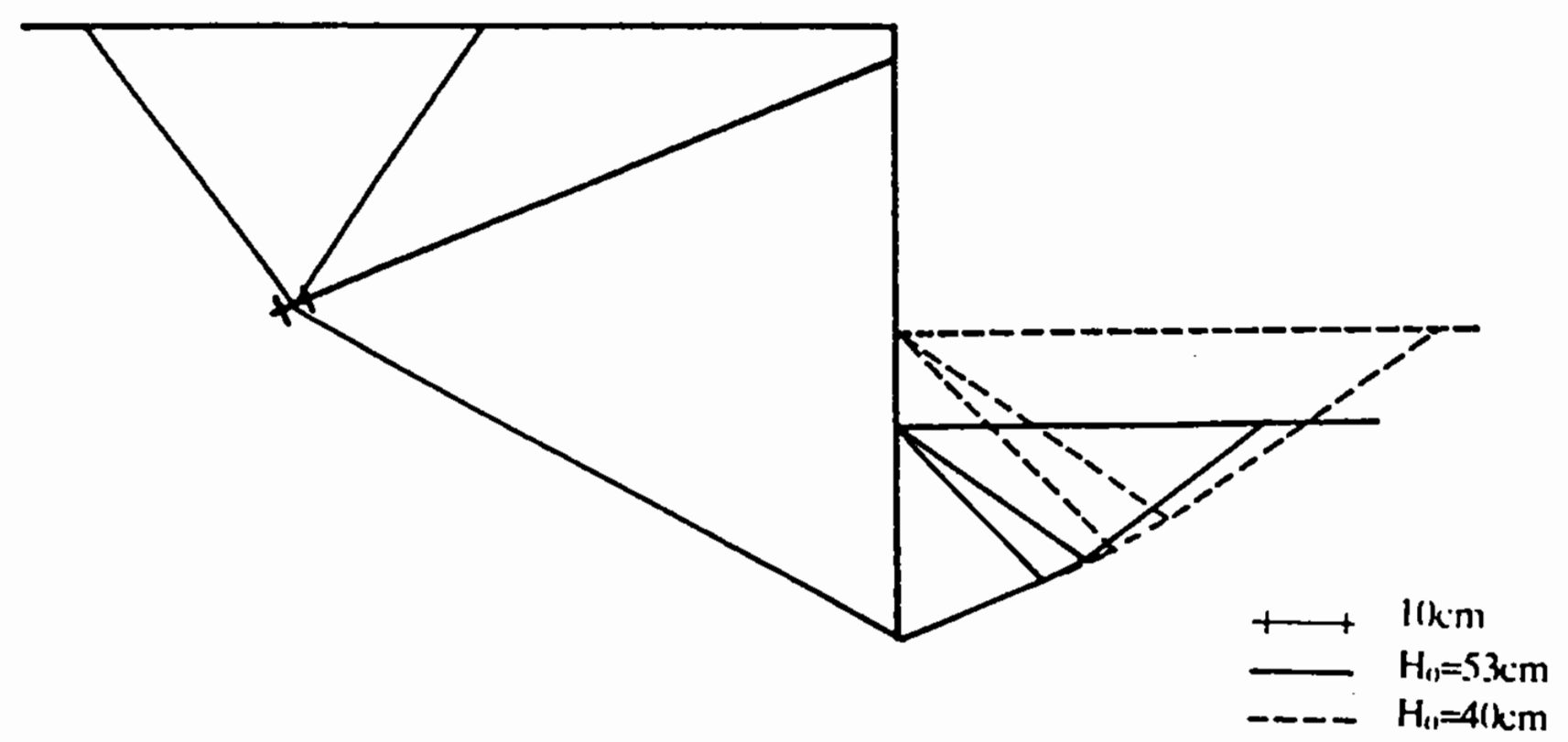

Figure 9. Influence de la profondeur d'excavation sur la surface de rupture critique. Influence of the excavation depth on the failure surface 


\subsection{Influence de la longueur d'ancrage et de son inclinaison}

La figure 10 présente la variation des facteurs $F_{s}$ et $F_{c}$ en fonction de la longueur d'ancrage $\mathrm{l}\left(\mathrm{H}_{0}=50,5 \mathrm{~cm} ; \alpha=22^{\circ}\right)$. Ces deux facteurs augmentent avec la longueur d'ancrage.

Pour illustrer l'influence de la longueur d'ancrage sur la surface de rupture la plus critique, nous présentons sur la figure 11 les surfaces critiques pour deux valeurs de la longueur d'ancrage $(1=75 \mathrm{~cm}$ et $100 \mathrm{~cm})$. Pour $\mathrm{l}=75 \mathrm{~cm}$, la rupture est imminente $\left(F_{s}=F_{c}=1\right)$, alors que pour $\mathrm{l}=100 \mathrm{~cm}$, on a $F_{c}=2,07$ et $F_{s}=1,24$. D'autre part, les angles $\theta_{1}$ et $\theta_{2}$ obtenus à partir de la minimisation numérique sont toujours égaux à $\pi / 4+\varphi / 2$. Quant à la zone passive, les angles $\theta_{3}$ et $\theta_{4}$ varient de telle manière que le prisme CIJ est toujours en état d'équilibre limite de Rankine.

La figure 12 montre la variation des facteurs $F$, et $F_{c}$ en fonction de l'inclinaison $\alpha$ du tirant d'ancrage $\left(\mathrm{H}_{0}=49 \mathrm{~cm} ; \mathrm{l}=75 \mathrm{~cm}\right)$.

Ces deux facteurs augmentent avec l'inclinaison de l'ancrage. Pour montrer l'influence de l'inclinaison de l'ancrage sur la surface de rupture la plus critique, nous présentons sur la figure 13 les surfaces critiques pour deux valeurs de l'inclinaison $\alpha\left(\alpha=12^{\circ}\right.$ et $\left.35^{\circ}\right)$. Pour $\alpha=12^{\circ}$, la rupture est imminente $\left(F_{s}=F_{c}=1\right)$, alors que pour $\alpha=35^{\circ}$, on a $F_{c}=1,25$ et $F_{s}=1,11$. Les conclusions précédentes concernant l'évolution des paramètres angulaires $\theta_{1}, \theta_{2}, \theta_{3}$ et $\theta_{4}$ restent identiques dans ce cas.

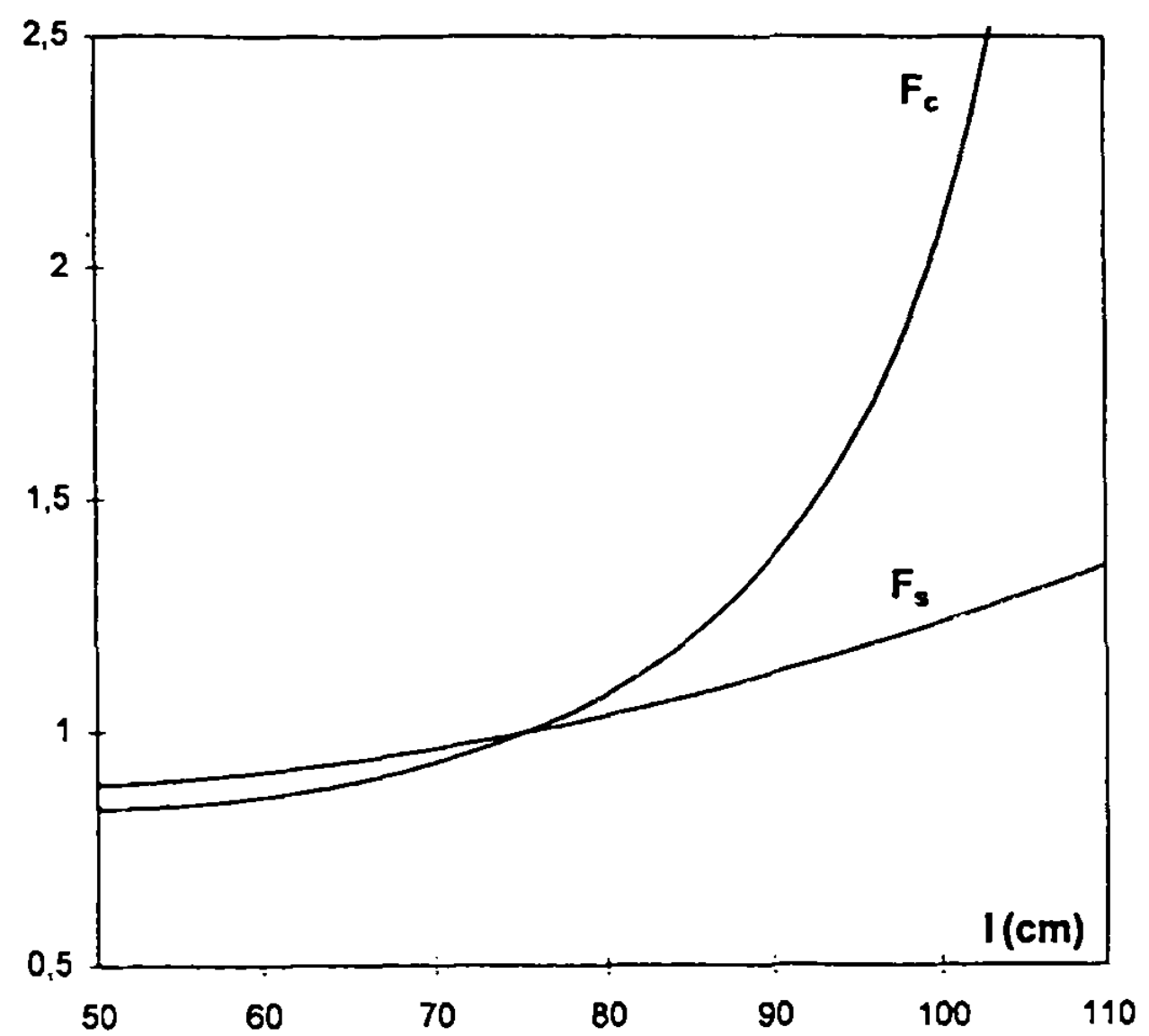

Figure 10. Variation des facteurs de sécurité $F_{s}$ et $F_{c}$ avec la longueur de l'ancrage. $F_{s}$ and $F_{c}$ versus the anchor length 


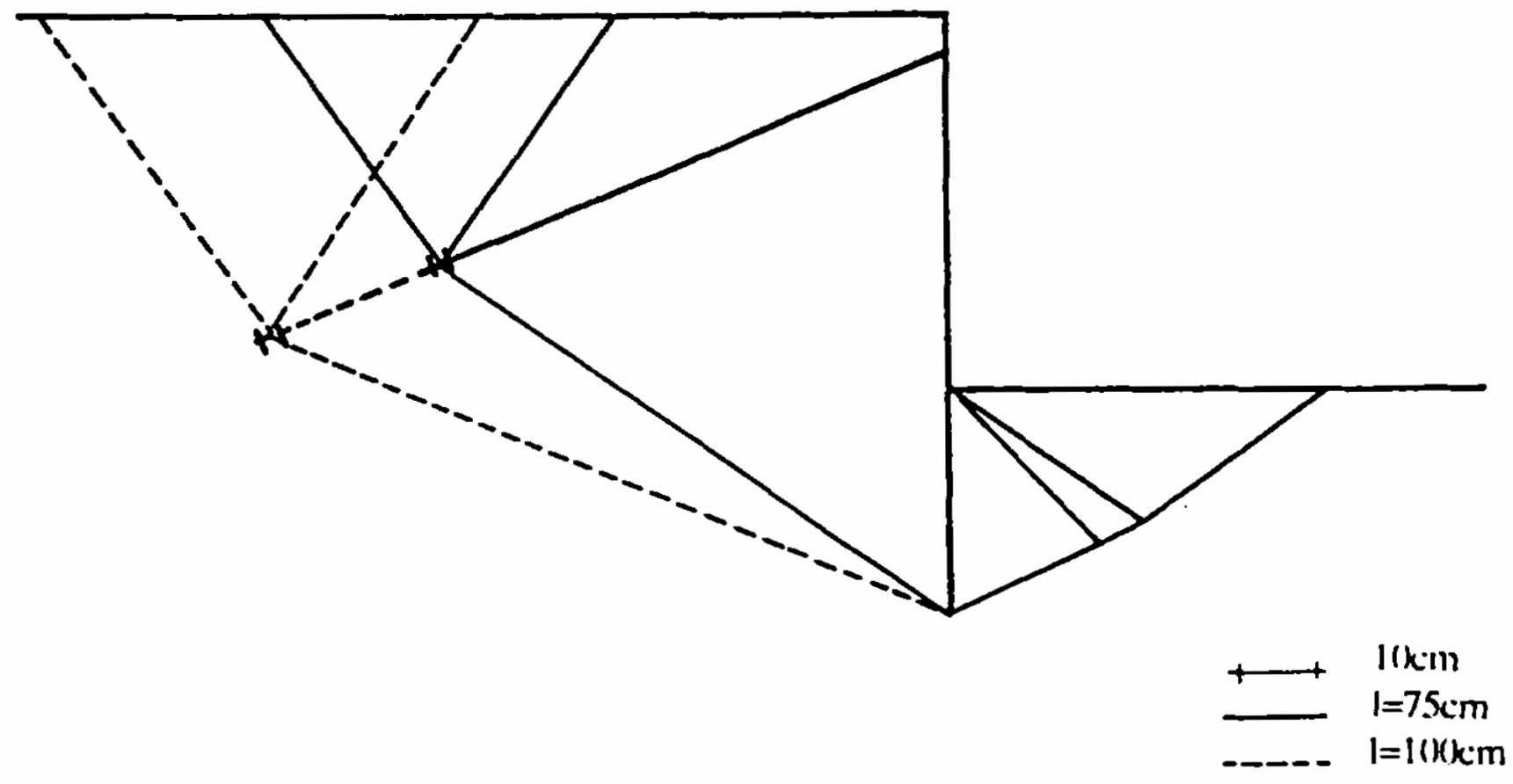

Figure 11. Influence de la longueur de l'ancrage sur la surface de rupture critique. Influence of the anchor length on the failure surface

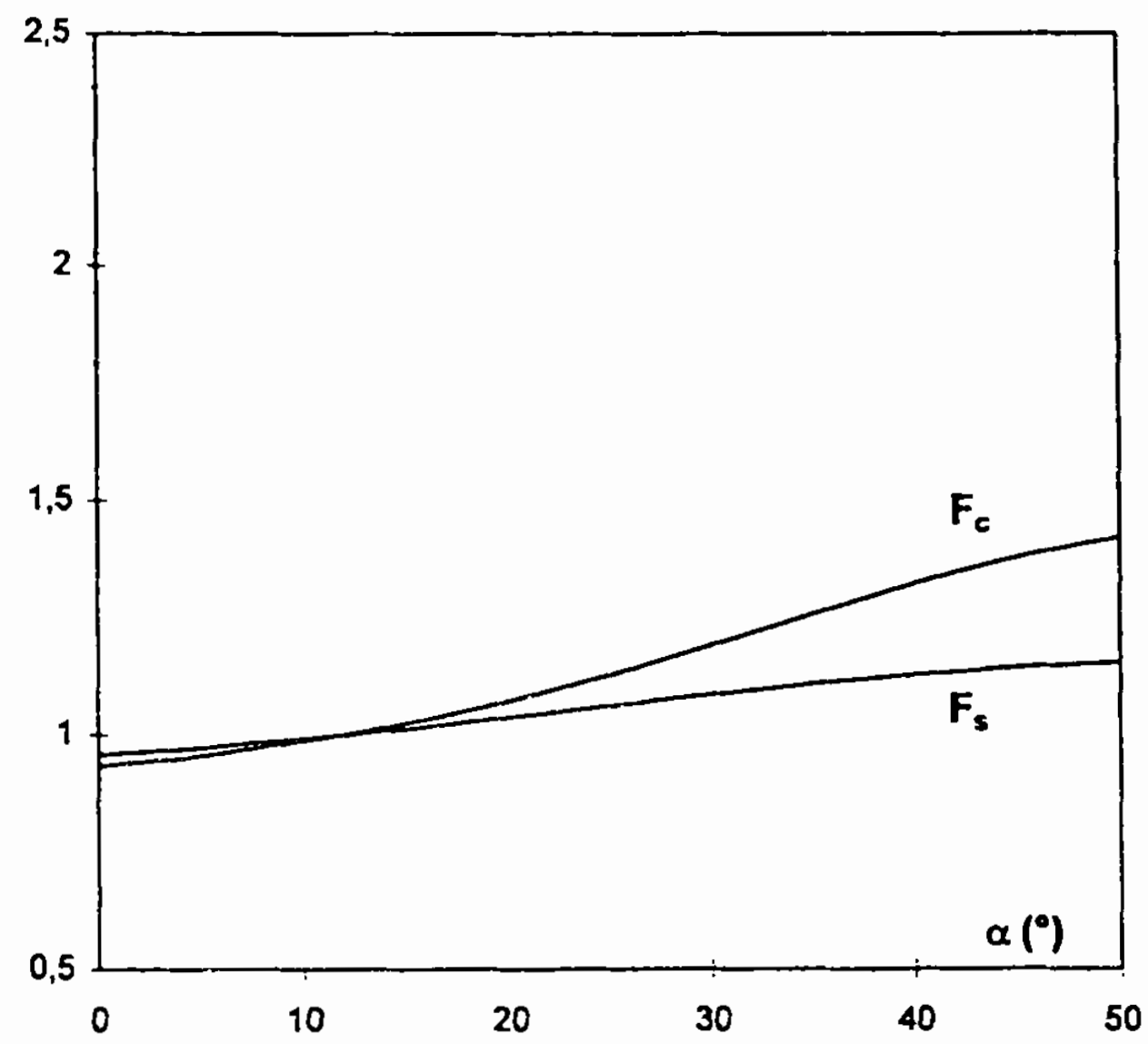

Figure 12. Variation des facteurs de sécurité $F_{s}$ et $F_{c}$ avec l'inclinaison de l'ancrage. $\mathrm{F}_{\mathrm{s}}$ and $\mathrm{F}_{\mathrm{c}}$ versus the anchor inclination 


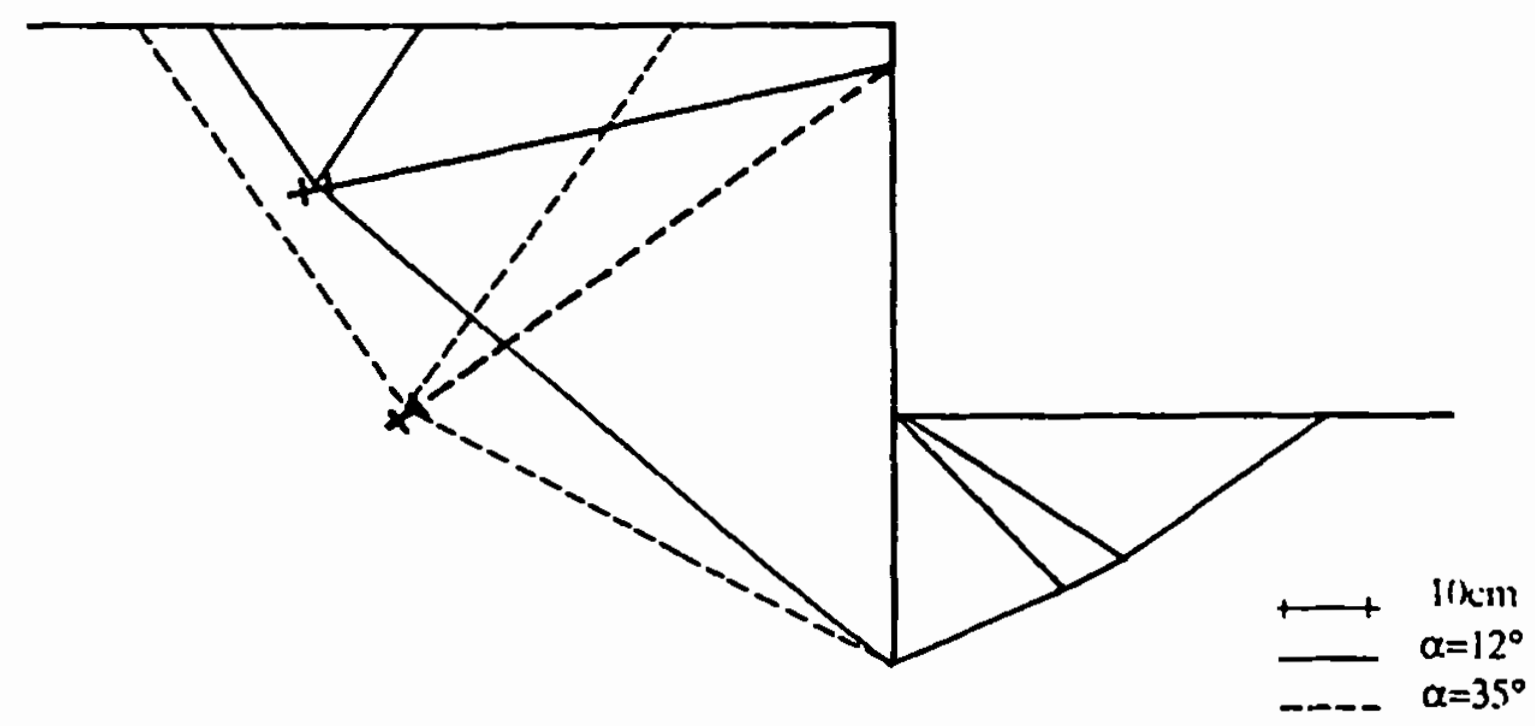

Figure 13. Influence de l'inclinaison de l'ancrage sur la surface de rupture critique. Influence of the anchor inclination on the failure surface

\subsection{Confrontation avec les résultats expérimentaux}

Le calcul classique par la méthode cinématique de la théorie de l'analyse limite est basé sur l'hypothèse d'une loi associée où $\psi=\varphi$. Cela se traduit par un comportement fortement dilatant du sol. Pour les sols réels, les observations expérimentales ont montré que le sol est un matériau non associé caractérisé par un angle de dilatance $\psi$ souvent bien plus faible que $\varphi$. Une étude récente présentée par Drescher et Detournay [DRE 93] a montré qu'il est possible d'introduire le caractère non associé de la déformation du sol dans l'étude des mécanismes de rupture translationnels. Le calcul théorique dans le cas de sols réels non associés peut alors être mené comme dans le cas des matériaux associés à condition de remplacer $c$ et $\varphi$ par $c^{*}$ et $\varphi^{*}$ donnés par :

$$
\begin{aligned}
& \tan \varphi^{\bullet}=\frac{\cos \psi \sin \varphi}{1-\sin \psi \sin \varphi} \\
& c^{\bullet}=\frac{\cos \psi \cos \varphi}{1-\sin \psi \sin \varphi} c
\end{aligned}
$$

Dans le cas de notre modèle réduit, l'angle de dilatance $\psi$ du matériau analogique de Schneebeli est également inférieur à $\varphi$. La valeur de $\psi$ mesurée à partir d'essais de compression biaxiaux se situant autour de $5^{\circ}$. Nous présentons dans le tableau 3 
les valeurs des profondeurs d'excavation critiques correspondant à la rupture imminente obtenues d'une part, à partir des essais expérimentaux sur le modèle réduit, et d'autre part, à partir du modèle théorique en analyse limite en considérant tout d'abord un matériau associé $(\psi=\varphi)$, puis en remplaçant $\varphi$ par $\varphi^{*}$ afin de prendre en compte la dilatance réelle du matériau.

\begin{tabular}{|c|c|c|c|c|c|c|c|c|c|}
\hline \multirow[b]{2}{*}{ Essai } & \multirow[b]{2}{*}{$1(\mathrm{~m})$} & \multirow[b]{2}{*}{$\begin{array}{c}\alpha \\
\left({ }^{\circ}\right)\end{array}$} & \multicolumn{7}{|c|}{ Profondeurs d'excavation critiques $\mathrm{H}_{0}(\mathrm{~m})$} \\
\hline & & & $\begin{array}{l}\text { Modèle } \\
\text { réduit }\end{array}$ & $\begin{array}{c}\text { Aralyse } \\
\text { limite } \\
\delta=8^{\circ} \\
\psi=\varnothing\end{array}$ & $\begin{array}{c}\text { Antyse } \\
\text { impe } \\
\delta=8^{\circ} \\
\psi=5^{\circ}\end{array}$ & $\begin{array}{c}\text { Ecart } \\
\text { mesure } \\
\text {-calcul } \\
(\%)\end{array}$ & $\begin{array}{c}\text { Arabse } \\
\text { limite } \\
\delta=14^{\circ} \\
\psi=\varphi\end{array}$ & $\begin{array}{c}\text { Arabse } \\
\text { limie } \\
\delta=14^{\circ} \\
\psi=5^{\circ}\end{array}$ & $\begin{array}{c}\text { Ecart } \\
\text { mesure } \\
\text {-calcul } \\
(\%)\end{array}$ \\
\hline Al & 0,850 & 22 & 0,505 & 0,530 & 0,517 & 2,4 & 0,548 & 0,535 & 5,9 \\
\hline A2 & 0,750 & 22 & 0,490 & 0,500 & 0,491 & 0,2 & 0,524 & 0,512 & 4,5 \\
\hline A3 & 0,650 & 22 & 0,470 & 0,480 & 0,474 & 0,8 & 0,509 & 0,498 & 6,0 \\
\hline A4 & 0,550 & 22 & 0,425 & 0,470 & 0,464 & 9,2 & 0,500 & 0,491 & 15,5 \\
\hline A5 & 0,550 & 31 & 0,450 & 0,475 & 0,466 & 3,6 & 0,501 & 0,492 & 9,3 \\
\hline A6 & 0,750 & 31 & 0,510 & 0,520 & 0,504 & $-1,2$ & 0,537 & 0,523 & 2,5 \\
\hline
\end{tabular}

Tableau 3. Profondeurs d'excavation critiques. Critical excavation depths

Les calculs ont été menés ici avec deux valeurs de l'inclinaison relative des contraintes au contact sol-écran $\delta$. La première valeur de $\delta$, égale à $8^{\circ}$, correspond aux mesures de frottement effectuées par essai de cisaillement direct, la demi-boîte inférieure étant remplacée par l'écran. La seconde égale à $14^{\circ}$ correspond aux valeurs maximales observées lors d'essais de poussée-butée par translation réalisés avec un écran rigide.

La prise en compte de la dilatance réelle du matériau réduit légèrement les profondeurs d'excavation critiques, le pourcentage de réduction n'excèdant pas $3,1 \%$. Si l'on excepte les essais 4 et 5 réalisés avec l'ancrage le plus court, on constate que l'écart entre la valeur mesurée et la valeur calculée en considérant l'angle fictif $\varphi^{*}$ n'excède jamais $6 \%$ et reste inférieur à $3 \%$ avec l'hypothèse $\delta=8^{\circ}$ (tableau 3). Les écarts les plus importants observés lors des essais 4 et 5 peuvent être expliqués par un changement de mécanisme. Avec un ancrage très court, la rupture s'accompagne d'une rotation autour de la fiche, comme dans le cas d'un écran non ancré. On peut noter qu'une telle configuration est irréaliste dans la pratique, l'ancrage étant alors de longueur trop réduite pour être efficace. 


\section{Conclusion}

L'analyse de la rupture d'ensemble des rideaux ancrés montre que les méthodes de calcul basées sur l'approche de Kranz conduisent à des valeurs de l'effort critique d'ancrage parfois très sous-estimées par rapport à celles mesurées sur un modèle réduit. L'analyse de ces écarts montre que l'approche de Kranz est sensible essentiellement à la valeur de la poussée au contact sol-écran. Cette poussée diffère souvent sensiblement de la répartition théorique de pression active en raison de la courbure du rideau et des effets de voute qui provoquent des redistributions importantes de la pression. Si l'on examine le mécanisme de rupture mis en évidence par l'étude stéréophotogrammétrique, il apparaît clairement que l'effort d'ancrage est en réalité un effort interne au mécanisme de rupture. Un schéma de calcul prenant en compte l'ensemble des zones affectées par la rupture semble ainsi plus pertinent.

C'est pour cette raison que nous proposons une approche en analyse limite par la méthode cinématique. Le schéma de rupture retenu est déduit du mécanisme de rupture observé par stéréophotogrammétrie. Les résultats obtenus par cette approche sont en bon accord avec ceux observés lors des essais sur modèle, à l'exception des configurations où l'ancrage est très court, provoquant une rupture par basculement du rideau. De telles configurations doivent être évitées dans la pratique.

Le modèle proposé, qui constitue ainsi une amélioration notable du schéma de Kranz, demanderait néanmoins à être qualifié également par confrontation à des observations sur ouvrages réels.

\section{Bibliographie}

[ABD 91] ABDI, R., PASTOR, J., "Limit loads of reinforced structures", Symposium Plasticity'91, Grenoble, 1991.

[ABD 94] ABDi, R., De Buran, P., PAstor J., "Calculation of the critical height of a homogenized reinforced soil wall : a numerical approach ", Int. J. for Numerical and Analytical Methods in Geomechanics, Vol. 18, pp. 485-505, 1994.

[ABD 97] ABDl, R., ShAHROUR, I., "Etude de la stabilité des murs en terre armée sous chargements sismiques", Revue Française de Génie Civil, Vol. 1, n 3, pp. 569-584, 1997.

[BAC 84] BACOT, J., KASTNER, R., MEMIER, A., " Recherche en laboratoire de mécanique des sols - une utilisation particulière de la photogrammétrie ", Bulletin SFPT, $n^{\circ} 94, \mathrm{pp}$. 15$29,1984$.

[BOT 80] Bottero, A., NĖGre, R., Pastor, J., Turgeman, S., " Finite element method and limit analysis theory for soil mechanics problems ", Computational Methods Appl. Mech. And Engng, Vol. 22, pp. 131-149, 1980. 
[BUR 95] BUREAUX SÉCURTTAS, "Recommandation concernant la conception, le calcul, l'exécution et le contrôle des tirants d'ancrage", Recommandations T.A.95, $4^{c}$ édition, Paris, 1995.

[CAM 75] CAMBEFoRT, H., "Parois de soutènement maintenues par une ligne d'ancrage ", Annales de l'Institut Technique du Bâtiment et des Travaux Publics, $\mathrm{n}^{\circ}$ 333, pp. 27-44, 1975.

[CHE 75] CHEN, W.F., Limit analysis and soil plasticity, Elsevier Scientific, London. 1975.

[DEB 89] de Buhan, P., Manglavacchi, R., Nova, R., Pellegrini, G. Salençon, J., "Yield design of reinforced earth walls by a homogenization method", Géotechnique, Vol. 39, n², pp. 189-201, 1989.

[DEB 91] DE BUhan, P., TALIERCIO., A., " A homogenization approach to the yield strength of composite materials ", Eur. J. Mech., A/Solids, 10, pp. 129-154, 1991.

[DEB 93] DE BUHAN, P., SALENÇON, J., "A comprehensive stability analysis of soil nailed structures ", Eur. J. Mech., A/Solids, Vol. 12, n³, pp. 325-345, 1993.

[KAS 82] KASTNER, R., " Excavations profondes en site urbain - Problèmes liés à la mise hors d'eau - Dimensionnement des soutènements butonnés ", Thèse de Docteur-èsSciences, INSA de Lyon et Université de Lyon 1, 409 p, 1982.

[KRA 53] KRANZ, E., Uber die verankerung von spundwande, 2nd edition, Wm. Ernst und sohn, Berlin, 1953.

[LIT 72] LITTEJoHN, G.S., " Anchored diaphragm walls in sand - Anchor design ", Ground Engineering, Vol. 5, $\mathrm{n}^{\circ}$ 1, pp. 12-17, 1972.

[MAS 86] MASROURI, F., "Comportement des rideaux de soutènement semi-flexibles : étude théorique et expérimentale ", Thèse de Doctorat, INSA de Lyon, 274 p, 1986.

[OST 77] Ostermayer, H., "Practice in the detail design application of anchorages ", A review of diaphragm walls, Institution of Civil Engineers, London, pp. 55-61, 1977.

[PAS 78] PASTOR, J., " Analyse limite : Détermination de solutions statiques complètes. Application au talus vertical ", Journal de Mécanique Appliquée, Vol. 2, n 2, pp. 167 197, 1978.

[ROW 72] RowE, P.W., " Earth pressure on flexible structures - Oral presentation ", Vème Congrès Européen de Mécanique des Sols et des Travaux de Fondation, Madrid, 1972.

[SAL 83] SAlençon, J., Calcul à la rupture et analyse limite, Presse de l'ENPC, Paris, $366 \mathrm{p}$.

[SAL 90] SALENÇON, J., " An introduction to the yield design theory and its application to soil mechanics ", Eur. J. Mech., A/Solids, Vol. 9, n 5, pp. 477-500, 1990.

[SAL 93] SALENÇON, J., "Yield design : a survey of the theory ", In evaluation of the global bearing capacities of structures, ed. By G. Sacchi-Landriani, J. Salençon, Springer Verlag, Wien, New York, 1993.

[SAL 95a] SAl.EnçON, J., PECKER, A., "Ultimate bearing capacity of shallow foundations under inclined and eccentric loads; Part 1 : purely cohesive soil ", European Journal of Mechanics, A/Solids, Vol. 14, n³, pp. 349-375, 1995. 
[SAL 95b] SAlençon, J., PECKER, A., "Ultimate bearing capacity of shallow foundations under inclined and eccentric loads; Part II : purely cohesive soil without tensile strength ", European Journal of Mechanics, A/Solids, Vol. 14, n³, pp. 377-396, 1995.

[SCH 57] SChNeEbel, G., "Une analogie mécanique pour l'étude de la stabilité des ouvrages en terre à deux dimensions ", IVe Congrès International de Mécanique des Sols et des Travaux de Fondation, vol. 2, Londres, pp. 228-232, 1957.

[SCH 76] SchulZ, H., "The definition of the factor of safety of multi-tied back walls", Proceedings of the 6th European Conference on Soil Mechanics and Foundation Engineering, Vol. 2, Vienna, pp. 189-196, 1976. 